Research Article

\title{
Pricing Catastrophe Equity Put Options in a Mixed Fractional Brownian Motion Environment
}

\author{
Guohe Deng \\ College of Mathematics and Statistics, Guangxi Normal University, Guilin 541004, China \\ Correspondence should be addressed to Guohe Deng; dengguohe@mailbox.gxnu.edu.cn
}

Received 25 March 2020; Accepted 23 April 2020; Published 11 May 2020

Guest Editor: Wenguang Yu

Copyright ( 2020 Guohe Deng. This is an open access article distributed under the Creative Commons Attribution License, which permits unrestricted use, distribution, and reproduction in any medium, provided the original work is properly cited.

\begin{abstract}
This paper considers the pricing of the CatEPut option (catastrophe equity put option) in a mixed fractional model in which the stock price is governed by a mixed fractional Brownian motion (mfBM model), which manifests long-range correlation and fluctuations from the financial market. Using the conditional expectation and the change of measure technique, we obtain an analytical pricing formula for the CatEPut option when the short interest rate is a deterministic and time-dependent function. Furthermore, we also derive analytical pricing formulas for the catastrophe put option and the influence of the Hurst index when the short interest rate follows an extended Vasicek model governed by another mixed fractional Brownian motion so that the environment captures the long-range dependence of the short interest rate. Based on the numerical experiments, we analyze quantitatively the impacts of different parameters from the mfBM model on the option price and hedging parameters. Numerical results show that the $\mathrm{mfBM}$ model is more close to the realistic market environment, and the CatEPut option price is evaluated accurately.
\end{abstract}

\section{Introduction}

As European put options, catastrophe equity put option (hereafter referred to as the CatEPut option) provides the insurance company the right to sell a specified amount of its stock to investors at a predetermined price at the expiration date of the option in case the total accumulated losses of the insured surpass a given trigger level of losses during the life time of the option. The CatEPut option is a catastrophe risk management tool and is firstly issued by RLI Corporation in 1996 to cover the potential losses caused by catastrophes such as typhoons, hurricanes, storms, waves, and earthquakes. Conceptually, CatEPut options and European put options are similar in that the two contractual financial instruments allow the holder special rights to sell securities. However, a CatEPut option differs from a European put option in two ways. One difference is that the CatEPut option is a sort of double trigger option. When the total accumulated losses surpass a given trigger level of losses during the lifetime of option, the CatEPut option is only exercisable. Other difference is that CatEPut options usually have five years or more to maturity. Consequently, the pricing mechanism for the CatEPut option is more complex.

There are few studies on pricing the CatEPut option in recent years. Cox et al. [1] first applied arbitrage-free approach to obtain a closed-form solution for the price of this option when the underlying asset price process satisfies the famous Black-Scholes model with the aggregate catastrophe losses of an insurance company following a Poisson process. Besides, they assumed that only the event of a catastrophe affects the underlying asset price, while the size of the catastrophe is irrelevant. Jaimungal and Wang [2] extended the work of Cox et al. [1] to analyze the pricing and hedging of the CatEPut option in a stochastic interest rate framework suggested by Vasicek [3] and assumed that the aggregate catastrophe losses followed a compound Poisson process and the underlying asset price process depended on the total loss level rather than on only the total number of losses. Furthermore, the underlying asset price process is modeled through a jump-diffusion process which is correlated to the loss process. Chang and Hung [4] supposed that the underlying asset price process was driven by a Lévy process 
with finite activity, and log jump sizes were independent identically distributed random variables with negative exponential density function and extended works of Cox et al. [1] and Jaimungal and Wang [2]. Lin et al. [5] considered different effects of catastrophe events and adopted a double Poisson process with log-normal intensity to model the number of catastrophe losses and derived the general pricing formula for contingent capital. Lin and Wang [6] applied a mixture of Erlangs as the distribution of the amounts for catastrophe losses and provided a pricing formula for the perpetual American CatEPut options using a penalty function approach. In addition, the pricing models for the CatEPut options with default risk have recently been studied (see Jiang et al. [7]; Jin and Zhong [8]; Koo and Kim [9] and $\mathrm{Xu}$ and Wang [10] for examples).

Recently, some researchers have adopted the stochastic volatility model or jump-diffusion model to describe the underlying asset price process. Kim et al. [11] extended the work of Lin and Wang [6] to the stochastic volatility model suggested by Heston [12] and investigated the value of the CatEPut options. Wang [13] presented CatEPut options with target variance which represents the insurance company's expectation of future realized variance under Heston's [12] model with stochastic interest rate and log-normal intensity of Poisson process. Wang et al. [14] considered pricing and hedging for the CatEPut options under a Markov-modulated jump-diffusion process with a Markov regime switching compensator. Kim et al. [15] provided a CatEPut option pricing model in which stock prices follow an additional jump term being moderately correlated with catastrophe losses and obtained a pricing formula for the perpetual American CatEPut options. Yu [16] and Yu et al. [17] studied pricing of the CatEPut options with double compound Poisson processes in which all jump terms are assumed to be independent mutually. The latest research studies on the valuing death benefits and population growth model were given by $\mathrm{Yu}$ et al. $[18,19]$ and Zhang et al. [20].

The aforementioned works have made significant contributions to the study of pricing catastrophe derivatives. However, none of the above works takes account of selfsimilarity and long-range dependence. A large number of empirical studies have shown that the distributions of the logarithm returns of the financial assets generally exhibit features of self-similarity and long-range dependence with heavy tails and volatility clustering in the real world (e.g., see Lo [21], Willinger et al. [22], Cont [23], Kang and Yoon [24], Kang et al. [25], and Huang et al. [26]). To incorporate this issue, the fractional Brownian motion (fBM) is introduced, which can capture the long-range dependence of the asset returns and produce burstiness in its sample path. Unfortunately, the $\mathrm{fBM}$ is neither a Markov nor a semimartingale and may, in some cases, still lead to implementable naive arbitrage opportunities (e.g., see Kuznetsov [27]; Bjork and Hult [28]). To get around this problem while taking into account the features of self-similarity and long-range dependence, the mixed fractional Brownian motion $(\mathrm{mfBM})$ is suggested, which is a linear combination of a Brownian motion (BM) and an $\mathrm{fBM}$ with Hurst index $H \in(1 / 2,1)$, to depict the underlying dynamics of the financial assets, and hence, it is arbitrage-free (see Cheridito [29, 30]). Recently, there has been considerable interest in applications of the $\mathrm{mfBM}$ environment on the pricing of various derivatives. One can refer to Deng and Xi [31]; Prakasa Rao [32]; Sun [33]; Xiao et al. [34, 35]; Zhang et al. [36]; He and Chen [37]; and Mehrdoust et al. [38] and the references therein. On the contrary, long-range dependence in bond market is a stylized fact that has been empirically studied by McCarthy et al. [39], Gil-Alana [40], Tabaka and Cajueiro [41], Cajueiro and Tabak [42], and Cajueiro and Tabak [43]. In this case, it is important to infer that there exists the long-range dependence in stochastic interest rates. Motivated by these results mentioned above, to make pricing models of financial derivatives more realistic, it is natural to replace the $\mathrm{BM}$ with the $\mathrm{mfBM}$ in stochastic interest rate models (see Xiao et al. [35]; Zhou et al. [44]).

In this paper, to price the CatEPut options under the stochastic interest rate framework and to capture the longrange dependence of both the underlying asset price and the short interest rate, we consider the pricing problem of this option in the mixed fractional Brownian motion environment with the mixed fractional Vasicek interest rate model. The contribution of this paper is two-fold. First, using the fractional Girsanov transform approach and the fractional Ito formula, we proposed the pricing models for the CatEPut options with constant or stochastic interest rates driven by the mixed fractional Vasicek model. Second, to assess the performance of our model, we apply the proposed model to compare the pricing results with those obtaining from traditional models, such as the famous Black-Scholes model and the $\mathrm{ABM}$ model.

The outline of this paper is as follows. Section 2 introduces the model for the $\mathrm{mfBM}$, the underlying asset, and the catastrophe losses. Section 3 derives the explicit pricing formulas for the CatEPut options with constant or stochastic interest rates driven by the fractional Vasicek model. In Section 4, we provide some numerical examples of option prices to compare the pricing results with those obtaining from the famous Black-Scholes and fBM models and to observe effects of model parameters. Section 5 presents the dynamical hedging strategies for the CatEPut options. Section 6 summarizes this paper and discusses possible directions for future research.

\section{The Model}

In the section, we briefly review some background concerning the mixed fractional Brownian motion and describe a mixed fractional Brownian motion with the jump environment.

Let $(\Omega, \mathscr{F}, Q)$ be a complete probability space equipped with a filtration $\left(\mathscr{F}_{t}\right)_{t \geq 0}$ satisfying the usual conditions. All the random processes in this paper are defined on this given probability space. A $\operatorname{mfBM} M_{t}^{H}$ of parameters $\alpha, \beta$, and $H$ is defined by a linear combination of a standard Brownian motion $W_{t}$ and an independent standard fractional Brownian motion $W_{t}^{H}$ with Hurst index $H(0<H<1)$, that is, $M_{t}^{H}=\alpha W_{t}+\beta W_{t}^{H}$, where $\alpha$ and $\beta$ are some real constants, not both are zero. 
According to the definition above, it is clear that the $\operatorname{mfBM} M_{t}^{H}$ nests some popular processes in the literature. The mfBM degenerates to the fBM with $\alpha=0$ and the BM with $\beta=0$. The process $M_{t}^{H}$ is a centered Gaussian process with $M_{0}^{H}=0$ and with mean 0 for all $t \geq 0$ and covariation function $\operatorname{Cov}\left(M_{t}^{H}, M_{s}^{H}\right)=\alpha^{2} \min \{t, s\}+\beta^{2} / 2$ $\left(|t|^{2 H}+|s|^{2 H}-|t-s|^{2 H}\right)$, for all $s, t \geq 0$. The increments of $M_{t}^{H}$ are positively correlated if $H \in((1 / 2), 1)$, uncorrelated if $H=1 / 2$, and negatively correlated if $H \in(0,(1 / 2))$. It is remarkable that the $\mathrm{mfBM} M_{t}^{H}$ is the standard $\mathrm{BM}$ if $H=1 / 2$, the increments of $M_{t}^{H}$ are long-range dependent if and only if $H \in((1 / 2), 1)$, and a self-similar process.

In order to ensure the market environment being arbitrage-free, we assume in this paper that the Hurst index $H \in((3 / 4), 1)$, which leads to the $\mathrm{mfBM}$ being equivalent to a Wiener process (see Cheridito [29]). Now, consider a frictionless and arbitrage-free financial market where information arrives both continuously and discontinuously, and there exists a underlying stock $S_{t}$ and a money market account $B_{t}$, which are traded continuously over a finite time interval $[0, T]$. Let $r_{t}$ be the instantaneous interest rate process and $L_{t}$ represent the total loss process of the insured over the time interval $[0, t]$, which is assumed to follow a compound Poisson process. Under the probability measure $Q$, the processes of $S_{t}, B_{t}$, and $L_{t}$ can be expressed as follows:

$$
\left\{\begin{array}{l}
S_{t}=S_{0} \exp \left\{-\mu L_{t}+\lambda \kappa t+X_{t}\right\}, \\
L_{t}=\sum_{i=1}^{N_{t}} Y_{i}, \\
B_{t}=B_{0} e^{\int_{0}^{t} r_{u} \mathrm{~d} u}, \quad B_{0}=1, \\
\mathrm{~d} X_{t}=\left[r_{t}-\frac{1}{2} \sigma_{s}^{2}\left(\alpha^{2}+2 \beta^{2} H t^{2 H-1}\right)\right] \mathrm{d} t+\sigma_{s} \mathrm{~d} M_{t}^{H},
\end{array}\right.
$$

where $\mu$ is a positive constant factor which denotes the percentage drop in the share value per unit loss, $N_{t}$ is a Poisson process with constant intensity $\lambda,\left\{Y_{i}\right\}_{i=1}^{\infty}$ denote the size of $i$-th catastrophe loss and form a sequence of independent identically distributed random variables with probability density function $f_{Y}(y)$, and $\sigma_{s}$ is the instantaneous volatility of the stock and is assumed to be constant. Additionally, all sources of randomness including the mfBM $M_{t}^{H}$, the Poisson process $N_{t}$, and the loss size sequence $\left(\left\{Y_{i}\right\}_{i=1}^{\infty}\right)$ are assumed to be mutually independent. We also define the filtration $\mathscr{F}_{t}$ generated by $\mathscr{F}_{t}=\mathscr{F}_{t}{ }_{t}$, where $\mathscr{F}_{t}{ }_{t}=$ $\sigma\left(S_{u}, u \leq t\right)$ is the natural filtration generated by $S_{t}$.

Since the share price of the underlying stock specified in (1) follows the compound Poisson process, the financial market is incomplete, and there is no unique equivalent martingale measure. We assume in this paper that the probability measure $Q$ is a risk-neutral probability measure provided by the agents, and the jump risk presents the nonsystematic and diversifiable risk (see, e.g., Cox et al. [1]; Jaimungal and Wang [2]). Hence, in order to ensure the discounted stock price is a $Q$-martingale, the parameter $\kappa$ satisfies $E\left(e^{-\mu L_{t}+\lambda \kappa t}\right)=1$; here, $E(\cdot)$ denotes the quasi-expectation under measure $Q$.

Remark 1. $E\left(e^{-\mu L_{t}+\lambda \kappa t}\right)=1 \Rightarrow \kappa=\int_{0}^{+\infty}\left(1-e^{-\mu y}\right) f_{Y}(y) \mathrm{d} y$. From (1), it is easy to know that

$$
\begin{aligned}
S_{T}= & S_{t} \exp \left\{\int_{t}^{T} r_{u} \mathrm{~d} u-\mu\left(L_{T}-L_{t}\right)+\lambda \kappa(T-t)\right. \\
& \left.-\frac{1}{2} \sigma_{s}^{2}\left[\alpha^{2}(T-t)+\beta^{2}\left(T^{2 H}-t^{2 H}\right)\right]+\sigma_{s}\left(M_{T}^{H}-M_{t}^{H}\right)\right\} .
\end{aligned}
$$

This implies that the stock price $S_{T}$ is log-normally distributed with $\ln S_{T} \sim N\left(m, v^{2}\right)$ under $r_{t}$ and $L_{t}$ known on the time interval $[t, T]$, where $m=\ln S_{t}+\int_{t}^{T} r_{u} \mathrm{~d} u-\mu\left(L_{T}-\right.$ $\left.L_{t}\right)+\lambda \kappa(T-t)-(1 / 2) \sigma_{s}^{2}\left[\alpha^{2}(T-t)+\beta^{2}\left(T^{2 H}-t^{2 H}\right)\right], v^{2}=$ $\sigma_{s}^{2}\left(\alpha^{2}(T-t)+\beta^{2}\left(T^{2 H}-t^{2 H}\right)\right)$, and $N\left(m, v^{2}\right)$ denotes the Gaussian distribution with mean $m$ and variance $v^{2}$.

\section{Pricing the CatEPut Option}

In this section, we shall first calculate the price of the CatEPut option when the short interest rate is a time-dependent and deterministic function. Second, we also derive a closed-form analytical expression for the price of the CatEPut option with the short interest rate following extended Vasicek's model in which the Brownian motion is replaced by a $\mathrm{mfBM}$ to capture the long-range dependence of the short-term interest rate.

The catastrophe put option is similar to a European put option, but it is termed a "double trigger" option, and its payoff at maturity $T$ is given by

$$
\text { payoff }=\left(K-S_{T}\right)^{+} 1\left(L_{T}-L_{t_{0}}>L\right),
$$

where $x^{+}=\max \{x, 0\}, K$ is the strike price, $L_{T}-L_{t_{0}}$ is the total accumulated losses of the insured over time period $\left(t_{0}, T\right], L$ is the trigger level of losses, and $1_{(\cdot)}$ denotes the indicator function. Let $P_{\text {cat }}\left(t, t_{0}\right)$ denote the CatEPut option price at time $t$, which was signed at time $t_{0}<t$ and matures at time $T>t$. In terms of the derivative pricing theory, we know that

$$
P_{\text {cat }}\left(t, t_{0}\right)=E\left[e^{-\int_{t}^{T} r_{u} \mathrm{~d} u}\left(K-S_{T}\right)^{+} 1_{\left(L_{T}-L_{t_{0}}>L\right)} \mid \mathscr{F}_{t}\right] .
$$

Proposition 1. Let the dynamics processes $S_{t}$ and $L_{t}$ be given by (1). When the short interest rate $r_{t}$ is a time-dependent and deterministic function, then the analytical pricing formula of the CatEPut option is

$$
P_{\text {cat }}\left(t, t_{0}\right) \sum_{n=1}^{\infty} \frac{[\lambda(T-t)]^{n} e^{-\lambda(T-t)}}{n !} \int_{\tilde{L}}^{+\infty} \Psi(y) f_{Y}^{(n)}(y) \mathrm{d} y,
$$

where $\widetilde{L}=\max \left\{0, L+L_{t_{0}}-L_{t}\right\}$, 


$$
\begin{aligned}
\Psi(y) & =K e^{-\int_{t}^{T} r_{u} \mathrm{~d} u} \Phi\left(-d_{2}(y)\right)-S_{t} e^{-\mu y+\lambda \kappa(T-t)} \Phi\left(-d_{1}(y)\right) \\
d_{1}(y) & =\frac{\ln \left(S_{t} / K\right)-\mu y+\lambda \kappa(T-t)+\int_{t}^{T} r_{u} \mathrm{~d} u+1 / 2 \sigma_{s}^{2}\left[\alpha^{2}(T-t)+\beta^{2}\left(T^{2 H}-t^{2 H}\right)\right]}{\sigma_{s} \sqrt{\alpha^{2}(T-t)+\beta^{2}\left(T^{2 H}-t^{2 H}\right)}} \\
d_{2}(y) & =d_{1}(y)-\sigma_{s} \sqrt{\alpha^{2}(T-t)+\beta^{2}\left(T^{2 H}-t^{2 H}\right)}
\end{aligned}
$$

and $f_{Y}^{(n)}(y)$ is the $n$-fold convolution of the loss probability density function $f_{Y}(y)$ and $\Phi(\cdot)$ denotes the cumulated standard normal distribution function.

Proof. See Appendix A.

Since the life time of the CatEPut option can be 5 years or more, we generalize the price formula obtained above to the stochastic interest rate framework. Jaimungal and Wang [2], Jiang et al. [7], Yu [16], Wang [13], Koo and Kim [9], and Xu and Wang [10] extend the results in Cox et al. [1] by incorporating stochastic interest rate, which is assumed to satisfy Vasicek's model in which the driving process is supposed to be a Brownian motion. Note that a substantial amount of empirical evidence suggests that the process of the short interest rate may exhibit long-memory and selfsimilarity behaviors. Similar to Xiao et al. [35] and Zhou et al. [44], we assume that the short interest rate is controlled by the following process under the risk-neutral pricing measure Q:

$$
\mathrm{d} r_{t}=a\left(b-r_{t}\right) \mathrm{d} t+\sigma_{r} \mathrm{~d} Z_{t}^{H}
$$

where $a$ denotes the mean-reversion rate, $b$ represents the long-run mean of the interest rate, $\sigma_{r}$ is the instantaneous volatility of the interest rate, and $Z_{t}^{H}=\alpha B_{t}+\beta B_{t}^{H}$ is another mfBM with parameters $\alpha, \beta$, and $H$ defined on the probability space $(\Omega, \mathscr{F}, Q)$ and is independent of the catastrophe loss process $L_{t}$. Moreover, the $\operatorname{mfBM} Z_{t}^{H}$ is correlated to the $\operatorname{mfBM} M_{t}^{H}$ with correlation coefficient $\rho \in[-1,1]$, which is given by $\mathrm{d} M_{t}^{H} \mathrm{~d} Z_{t}^{H}=\rho\left(\alpha^{2} \mathrm{~d} t+\beta^{2} \mathrm{~d} t^{2 H}\right)$. Additionally, we assume that $a, b, \sigma_{r}, \rho$ and the initial value $r_{0}$ of $r_{t}$ are real constants, standard Brownian motions, $W_{t}$ and $B_{t}$, are independent of the fractional Brownian motions, $W_{t}^{H}, B_{t}^{H}$, and the filtration $\mathscr{F}_{t}$ generated by $\mathscr{F}_{t}=\mathscr{F}_{t}{ }_{t} \vee \mathscr{F}_{t}^{r}$, where $\mathscr{F}_{t}^{r}=$ $\sigma\left(r_{u}, u \leq t\right)$ is the natural filtration generated by $r_{t}$.

Now, let us consider a zero-coupon bond that pays 1 unit of cash at maturity $T$. According to the risk-neutral pricing formula, the value of this bond at time $t \in[0, T]$, denoted by $P(t, T)$, is

$$
P(t, T)=E\left[e^{-\int_{t}^{T} r_{u} \mathrm{~d} u} \mid \mathscr{F}_{t}^{r}\right]
$$

Using the fractional Ito formula (see $\mathrm{Hu}$ and Oksendal [45]), we have

$$
\frac{\mathrm{d} P(t, T)}{P(t, T)}=r_{t} \mathrm{~d} t+\sigma_{r} \frac{\partial P / \partial r}{P(t, T)} \mathrm{d} Z_{t}^{H}
$$

Moreover, $P(t, T)$ satisfies the following P.D.E.:

$$
\left\{\begin{array}{l}
\frac{\partial P}{\partial t}+\frac{1}{2} \sigma_{r}^{2}\left(\alpha^{2}+2 \beta^{2} H t^{2 H-1}\right) \frac{\partial^{2} P}{\partial r^{2}}+a(b-r) \frac{\partial P}{\partial r}-r P=0 \\
P(T, T)=1
\end{array}\right.
$$

Similar to Xiao et al. [35], we obtain the following.

Corollary 1. When the short interest rate follows (7), the price of the zero-coupon bond is given by

$$
P(t, T)=\exp \left\{A(t, T)-B(t, T) r_{t}\right\},
$$

where $B(t, T)=1-e^{-a(T-t)} / a$ and $A(t, T)=(b-$ $\left.\left(\alpha^{2} \sigma_{r}^{2} / 2 a^{2}\right)\right)[B(t, T)-(T-t)]-\left(\alpha^{2} \sigma_{r}^{2} / 4 a\right) B^{2}(t, T)+$ $H \beta^{2} \sigma_{r}^{2} \int_{t}^{T} u^{2 H-1} B^{2}(u, T) d u$.

Now, we introduce the Radon-Nikodym derivative as follows:

$$
\begin{aligned}
\left.\frac{\mathrm{d} Q^{T}}{\mathrm{~d} Q}\right|_{\mathscr{F}_{T}}= & \frac{e^{-\int_{t}^{T} r_{u} \mathrm{~d} u}}{P(t, T)} \\
= & \exp \left\{-\frac{1}{2} \int_{t}^{T} \sigma_{r}^{2} B^{2}(u, T)\left(\alpha^{2}+2 H \beta^{2} u^{2 H-1}\right) \mathrm{d} u\right. \\
& \left.-\int_{t}^{T} \sigma_{r} B(u, T) \mathrm{d} Z_{u}^{H}\right\} .
\end{aligned}
$$

Using the fractional Girsanov theorem (see $\mathrm{Hu}$ and Oksendal [45]), then $\widehat{M}_{t}^{H}=\alpha \widehat{W}_{t}+\beta \widehat{W}_{t}^{H}, \widehat{Z}_{t}^{H}=\alpha \widehat{Z}_{t}+\beta \widehat{Z}_{t}^{H}$ defined by

$$
\begin{gathered}
\widehat{M}_{t}^{H}=M_{t}^{H}+\int_{0}^{t} \rho \sigma_{r} B(u, T)\left(\alpha^{2}+2 H \beta^{2} u^{2 H-1}\right) \mathrm{d} u, \\
\widehat{Z}_{t}^{H}=Z_{t}^{H}+\int_{0}^{t} \sigma_{r} B(u, T)\left(\alpha^{2}+2 H \beta^{2} u^{2 H-1}\right) \mathrm{d} u,
\end{gathered}
$$

are $Q^{T}$-mfBMs with the correlation coefficient $\rho$, and under $Q^{T}$, 


$$
\begin{aligned}
\ln S_{T}= & \ln \frac{S_{t}}{P(t, T)}-\mu\left(L_{T}-L_{t}\right)+\lambda \kappa(T-t)-\frac{1}{2} \sigma^{2}(u) \\
& +\int_{t}^{T} \sigma_{s} \mathrm{~d} \widehat{M}_{t}^{H}+\int_{t}^{T} \sigma_{r} B(u, T) \mathrm{d} \widehat{Z}_{t}^{H},
\end{aligned}
$$$$
\int_{t}^{T} \sigma_{s} \mathrm{~d} \widehat{M}_{t}^{H}+\int_{t}^{T} \sigma_{r} B(u, T) \mathrm{d} \widehat{Z}_{t}^{H} \sim N\left(0, \theta^{2}(t, T)\right),
$$

where $\quad \theta^{2}(t, T)=\int_{t}^{T} \widetilde{B}(u, T)\left(\alpha^{2}+2 H \beta^{2} u^{2 H-1}\right) \mathrm{d} u \quad$ and $\widetilde{B}(u, T)=\sigma_{s}^{2}+2 \rho \sigma_{s} \sigma_{r} B(u, T)+\sigma_{r}^{2} B^{2}(u, T)$. Therefore, (4) can be rewritten as

$$
P_{\text {cat }}\left(t, t_{0}\right)=P(t, T) E^{T}\left\{1_{\left(L_{T}-L_{t_{0}}>L\right)}\left(K-S_{T}\right)^{+} \mid \mathscr{F}_{t}\right\},
$$

where $E^{T}\left[\mathscr{F}_{t}\right]$ denotes the quasi-conditional expectation under the $T$-forward measure $Q^{T}$.

Proposition 2. Let the dynamics processes $S_{t}, L_{t}$, and $r_{t}$ be given by (1) and (7); then, the analytical pricing formula of the CatEPut option is

$$
P_{\text {cat }}\left(t, t_{0}\right)=\sum_{n=1}^{\infty} \frac{[\lambda(T-t)]^{n} e^{-\lambda(T-t)}}{n !} \int_{\widetilde{L}}^{+\infty} \widetilde{\Psi}(y) f_{Y}^{(n)}(y) \mathrm{d} y,
$$

where $\widetilde{L}=\max \left\{0, L+L_{t_{0}}-L_{t}\right\}$,

$$
\begin{aligned}
& \widetilde{\Psi}(y)=\operatorname{KP}(t, T) \Phi\left(-\tilde{d}_{2}(y)\right)-S_{t} e^{-\mu y+\lambda \kappa(T-t)} \Phi\left(-\widetilde{d}_{1}(y)\right), \\
& \tilde{d}_{1}(y)=\frac{\ln \left(S_{t} / K P(t, T)\right)-\mu y+\lambda \kappa(T-t)+1 / 2 \theta^{2}(t, T)}{\theta(t, T)}, \\
& \tilde{d}_{2}(y)=\tilde{d}_{1}(y)-\theta(t, T) .
\end{aligned}
$$

Proof. See Appendix A.

Remark 2. When $(\alpha, \beta)=(1,0)$, then the result of Proposition 2 reduces to that of Jaimungal and Wang [2]. When $(\alpha, \beta)=(0,1)$, then the result of Proposition 2 is provided in the $\mathrm{fBM}$ environment. Therefore, the JW and $\mathrm{ABM}$ models are special cases of the mfBM model in this paper.

Remark 3. The integrations in the pricing model of equation (17), such as $\theta^{2}(t, T)=\int_{t}^{T} \widetilde{B}(u, T)\left(\alpha^{2}+2 H \beta^{2} u^{2 H-1}\right) \mathrm{d} u$, can be calculated using the Riemann integral.

Cox et al. [1] considered that the loss size conditional on a loss is fixed at $l$ and the trigger level is an integer multiple of the loss size, i.e., $L=c l$. The parameter $c$, here labeled the trigger ratio level, represents the ratio of the trigger level to the total expected loss. The probability density function $f_{Y}(y)$ od loss size $Y_{i}$ in this fixed loss case is a Dirac density $f_{Y}(y)=\delta(y-l)$, and then the following corollary is given.

Corollary 2. If the loss size $Y_{i}$ follows a Dirac density $f_{Y}(y)=\delta(y-l)$, the price function in (17) can be rewritten as follows:

$$
\begin{aligned}
P_{\text {cat }}\left(t, t_{0}\right)= & \sum_{n=\widehat{N}+1}^{\infty} \frac{[\lambda(T-t)]^{n} e^{-\lambda(T-t)}}{n !}\left[K P(t, T) \Phi\left(-\widehat{\mathrm{d}}_{2}(n)\right)\right. \\
& \left.-S_{t} e^{-\mu n l+\lambda \kappa(T-t)} \Phi\left(-\widehat{\mathrm{d}}_{1}(n)\right)\right]
\end{aligned}
$$

where $\widetilde{N}=\max \left\{0, c+N_{t_{0}}-N_{t}\right\}$ and

$$
\widehat{d}_{1}(n)=\frac{\ln \left(S_{t} / K P(t, T)\right)-\mu n l+\lambda \kappa(T-t)+(1 / 2) \theta^{2}(t, T)}{\theta(t, T)},
$$$$
\widehat{d}_{2}(n)=\widehat{d}_{1}(n)-\theta(t, T) .
$$

It is clear that pricing model (17) depends on Hurst parameter $H$. Hence, we present the influence of this parameter based on price formulas (17) in the following proposition.

Proposition 3. The influence of the Hurst parameter $H$ is given by

$$
\begin{aligned}
\frac{\partial P_{\text {cat }}\left(t, t_{0}\right)}{\partial H}= & K P(t, T) \beta^{2} \sum_{n=1}^{\infty} \frac{[\lambda(T-t)]^{n} e^{-\lambda(T-t)}}{n !} \int_{\tilde{L}}^{+\infty} \int_{t}^{T} \\
& \cdot\left[\sigma_{r}^{2} \Phi\left(-\tilde{d}_{2}(y)\right) B^{2}(u, T)+\frac{\widetilde{B}(u, T) e^{-\left(\widetilde{d}_{2}^{2}(y) / 2\right)}}{\sqrt{2 \pi} \theta(t, T)}\right.
\end{aligned}
$$

$$
\left.\cdot[u+H(2 H-1)] u^{2 H-2} f_{Y}^{(n)}(y) \mathrm{d} u \mathrm{~d} y\right] .
$$

Moreover, we have $\left(\partial P_{c a t}\left(t, t_{0}\right) / \partial H\right)>0$ for fixed $t \in[0, T]$.

Proof. We get from (17) that

$$
\frac{\partial P_{\text {cat }}\left(t, t_{0}\right)}{\partial H}=\sum_{n=1}^{\infty} \frac{[\lambda(T-t)]^{n} e^{-\lambda(T-t)}}{n !} \int_{\tilde{L}}^{+\infty} \frac{\partial \widetilde{\Psi}(y)}{\partial H} f_{Y}^{(n)}(y) \mathrm{d} y .
$$

Since 


$$
\begin{aligned}
\frac{\partial \widetilde{\Psi}(y)}{\partial H} & =K \frac{\partial P(t, T)}{\partial H} \Phi\left(-\widetilde{d}_{2}(y)\right)-K P(t, T) \Phi^{\prime}\left(-\widetilde{d}_{2}(y)\right) \frac{\partial \widetilde{d}_{2}(y)}{\partial H}+S_{t} e^{-\mu y+\lambda \kappa(T-t)} \Phi^{\prime}\left(-\widetilde{d}_{1}(y)\right) \frac{\partial \widetilde{d}_{1}(y)}{\partial H} \\
\frac{\partial P(t, T)}{\partial H} & =P(t, T) \frac{\partial A(t, T)}{\partial H} \\
& =P(t, T) \beta^{2} \sigma_{r}^{2} \int_{t}^{T}[u+H(2 H-1)] B^{2}(u, T) u^{2 H-2} \mathrm{~d} u, \\
\frac{\partial \tilde{d}_{2}(y)}{\partial H} & =\frac{\partial \widetilde{d}_{1}(y)}{\partial H}-\frac{\partial \theta(t, T)}{\partial H} .
\end{aligned}
$$

On the contrary, we have

$$
\begin{aligned}
\frac{\partial \tilde{d}_{1}(y)}{\partial H} & =\frac{[-(1 / P(t, T))(\partial P(t, T) / \partial H)+\theta(t, T)(\partial \theta(t, T) / \partial H)]-\tilde{d}_{1}(y)(\partial \theta(t, T) / \partial H)}{\theta(t, T)}, \\
\frac{\partial \theta(t, T)}{\partial H} & =\beta^{2} \int_{t}^{T} \widetilde{B}(u, T)\left[\frac{u+t H n(2 H-))] u^{2 H-2} \mathrm{~d} u}{\theta(t, T) .}\right.
\end{aligned}
$$

With tedious calculation, we obtain that

$$
\begin{aligned}
\frac{\partial \widetilde{\Psi}(y)}{\partial H}= & K P(t, T) \beta^{2} \int_{t}^{T}\left[\sigma_{r}^{2} \Phi\left(-\widetilde{d}_{2}(y)\right) B^{2}(u, T)\right. \\
& \left.+\frac{\widetilde{B}(u, T) e^{-\widetilde{d}_{2}^{2}(y) / 2}}{\sqrt{2 \pi} \theta(t, T)}[u+H(2 H-1)] u^{2 H-2} \mathrm{~d} u\right] \\
& +\frac{\beta^{2}}{\sqrt{2 \pi}}\left[S_{t} e^{-\mu y+\lambda \kappa(T-t)} e^{-\left(\widetilde{d}_{1}^{2}(y) / 2\right)}-K P(t, T) e^{-\left(\widetilde{d}_{1}^{2}(y) / 2\right)}\right] \\
& \cdot \int_{t}^{T}\left[-\sigma_{r}^{2} B^{2}(u, T)+\left(1-\frac{\widetilde{d}_{1}(y)}{\theta(t, T)}\right) \widetilde{B}(u, T)\right] \\
& \cdot[u+H(2 H-1)] u^{2 H-2} \mathrm{~d} u .
\end{aligned}
$$

Note that $e^{-\left(\vec{d}_{2}^{2}(y) / 2\right)}=e^{-\left(\vec{d}_{1}^{2}(y) / 2\right)+\tilde{d}_{1}^{2}(y) \theta(t, T)-\left(\theta^{2}(t, T) / 2\right)}=$ $\left(S_{t} / K P(t, T)\right) e^{-\mu y+\lambda \kappa(T-t)} e^{-\widetilde{d}_{1}^{2}(y) / 2}$; then, (25) can be rewritten as

$$
\begin{aligned}
\frac{\partial \widetilde{\Psi}(y)}{\partial H}= & K P(t, T) \beta^{2} \int_{t}^{T}\left[\sigma_{r}^{2} \Phi\left(-\widetilde{d}_{2}(y)\right) B^{2}(u, T)\right] \\
& +\frac{\widetilde{B}(u, T) e^{-\tilde{d}_{2}(y) / 2}}{\sqrt{2 \pi} \theta(t, T)}[u+H(2 H-1)] u^{2 H-2} \mathrm{~d} u .
\end{aligned}
$$

Substituting (26) into (22) leads to finish the proof.
Corollary 4. The risk-neutral probability that the CatEPut option ends in the money under the dynamics processes $S_{t}, L_{t}$, and $r_{t}$ given by (1) and (7) is

$$
\begin{gathered}
Q\left(S_{T}<K, L_{T}-L_{t_{0}}>L \mid \mathscr{F}_{t_{0}}\right)=\sum_{n=1}^{\infty} \frac{\left[\lambda\left(T-t_{0}\right)\right]^{n} e^{-\lambda\left(T-t_{0}\right)}}{n !} \\
\int_{L}^{+\infty} \Phi(-h(y)) f_{Y}^{(n)}(y) \mathrm{d} y,
\end{gathered}
$$

where $\quad h(y)=\left(\ln \left(S_{t_{0}} / K P\left(t_{0}, T\right)\right)-\mu y+\lambda \kappa\left(T-t_{0}\right)-1 /\right.$ $\left.2 \theta^{2}\left(t_{0}, T\right)\right) /\left(\theta\left(t_{0}, T\right)\right)$.

Proof. Similar to the proof of $\widehat{I}_{1}$ in (B.2), it is obvious to obtain (27).

Although it appears that the pricing formula in (17) involves infinite sum, in practice, it is not necessary. In the following, we will prove that numerically, only the first few terms in the series are needed when the jump intensity rate $\lambda$ is small. Similar to the argument of Jaimungal and Wang [2], we let

$$
P_{\text {cat }}\left(t, t_{0} ; M\right)=\sum_{n=1}^{M} \frac{[\lambda(T-t)]^{n} e^{-\lambda(T-t)}}{n !} \int_{\widetilde{L}}^{+\infty} \widetilde{\Psi}(y) f_{Y}^{(n)}(y) \mathrm{d} y .
$$

Then, by (17), we have 


$$
\begin{aligned}
\left|P_{\text {cat }}\left(t, t_{0}\right)-P_{\text {cat }}\left(t, t_{0} ; M\right)\right| & =\sum_{n=M+1}^{\infty} \frac{[\lambda(T-t)]^{n} e^{-\lambda(T-t)}}{n !} \int_{\tilde{L}^{+\infty}}^{+\infty}(y) f_{Y}^{(n)}(y) \mathrm{d} y \\
& \leq \sum_{n=M+1}^{\infty} \frac{[\lambda(T-t)]^{n} e^{-\lambda(T-t)}}{n !} \int_{\tilde{L}^{+\infty}}^{+\infty} K(t, T) \Phi\left(-\widetilde{d}_{2}(y)\right) f_{Y}^{(n)}(y) \mathrm{d} y \\
& \leq \sum_{n=M+1}^{\infty} \frac{[\lambda(T-t)]^{n} e^{-\lambda(T-t)}}{n !} K P(t, T) \\
& \leq K P(t, T)\left\{\frac{[\lambda(T-t)]^{M}}{M !}\left(1-e^{-\lambda(T-t)}\right)\right\} .
\end{aligned}
$$

Consequently, the relative error in the price can be made very small by choosing $M$ appropriately. For example, if $\lambda=$ 0.5 and choosing $M=20$, an accuracy of $0.4 \%$ can be obtained.

\section{Numerical Results}

In this section, we analyze numerically the proposed model by considering the stochastic interest rate environment. First, we clarify the effectiveness of the proposed model by making comparison with the results of Jaimungal and Wang [2] and the fBM model. Second, we present a sensitivity analysis for the CatEPut option prices under alternative parameter values. In particular, we examine the effects of two parameters of the claim size on the option price and the riskneutral probability.

In the next numerical experiments, we consider the CatEPut option with maturity $T=5$ year and show some parameter values in Table 1 if there is no special instruction. The loss size in this paper follows the gamma distribution with mean $\mu_{l}$ and standard deviation $\sigma_{l}$, that is, $f_{Y}(y)=$ $\left(\left(\left(\mu_{l} / \sigma_{l}^{2}\right)^{\left(\mu_{l}^{2} / \sigma_{l}^{2}\right)}\right) /\left(\Gamma\left(\mu_{l}^{2} / \sigma_{l}^{2}\right)\right)\right) y^{\left(\mu_{l}^{2} / \sigma_{l}^{2}\right)-1} e^{-\left(\mu_{l} / \sigma_{l}^{2}\right) y}, y>0$, in which case, the $n$-fold convolution of the losses is given by

$$
f_{Y}^{(n)}(y)=\frac{\left(\mu_{l} / \sigma_{l}^{2}\right)^{\left(n \mu_{l}^{2} / \sigma_{l}^{2}\right)}}{\Gamma\left(n \mu_{l}^{2} / \sigma_{l}^{2}\right)} y^{\left(n \mu_{l}^{2} / \sigma_{l}^{2}\right)-1} e^{-\left(\mu_{l} / \sigma_{l}^{2}\right) y}, \quad y>0
$$

where $\Gamma(\cdot)$ is the gamma function. The trigger level of losses $L$ is defined by $c \lambda(T-t) \mu_{l}$ which denotes a multiple $c$ of the expected losses. According to Remark 1, the parameter $\kappa$ in this case is $\kappa=1-\left(\mu_{l} /\left(\mu_{l}+\mu \sigma_{l}^{2}\right)\right)^{\left(\mu_{l}^{2} / \sigma_{l}^{2}\right)}$. The parameter $c$ denotes the real-valued trigger ratio level. Moreover, we also assume $L_{t}-L_{t_{0}}=0$ and $(\alpha, \beta)=(1,1)$ for the mfBM.

Figure 1 displays all values of the CatEPut option against different values of $S_{0}$ with the trigger ratio level of $c=1$ and $c=2$ for three different models, including the JW (Jaimungal and Wang [2]), $\mathrm{fBM}$, and mfBM models in the stochastic interest rate framework.

As can be seen from Figure 1, the curvature of all option price curves with the trigger ratio level $c=1$ and $c=2$ for the three models is significantly decreased as the stock price increases. This shows that the value of $S_{0}$ has a negative effect on the value of the CatEPut option. In addition, the option prices for the JW model are lower than the corresponding prices for the $\mathrm{fBM}$ and $\mathrm{mfBM}$ models. We point that the difference is caused by the existence of the longrange dependence. It implies that the proposed model can not only exhibit the long-range dependence but also produce closer results to the JW model in which BM has been used traditionally as the driving force for modeling the stock price.

Table 2 reports the option prices for the in-the-money $\left(S_{0}=60\right)$, at-the-money $\left(S_{0}=80\right)$, and out-of-the-money $\left(S_{0}=100\right)$ CatEPut option under the constant/stochastic interest rate framework. As shown in Table 1, we know that the option price under stochastic interest rate is lower than the corresponding price under constant interest rate. This is reasonable because the introduction of stochastic interest rate reduces the variance of the return on the underlying stock compared with the situation with constant interest rate. No matter what the strike is, the price of the CatEPut option increases as the Hurst index $H$ and the stock's volatility $\sigma_{s}$ increase. One possible reason for this phenomenon is that the Hurst index represents the long-range dependency property. So, the bigger the Hurst index is, i.e., the stronger the long-memory property is, the higher the option price is. However, the option price decreases as the trigger ratio level $c$ increases, which is obvious. In addition, the CatEPut option's price decreases from in-the-money to out-of-the-money.

Next, we examine how the option price is affected by the model parameter values of the spot interest rate process for a trigger ratio level $c=1$. We focus on investigating the effects of two parameters $\sigma_{r}$ and correlation coefficient $\rho$ on the option price. The results are plotted in Figure 2.

Figure 2(a) indicates that the effect on option price is remarkable if the volatility of the spot interest rate is higher. This may be due to two facts: the uncertainty effect and the long-range dependence in the real world. The larger volatilities induce more uncertainty, and the long-range dependence produces burstiness in the sample path of asset returns, and these push the option prices upward. In Figure 2(b), we compare the option price with different 
TABLE 1: The parameter values used in numerical examples.

\begin{tabular}{lc}
\hline Parameter & Value \\
\hline$K$ & 80 \\
$r_{0}$ & 0.02 \\
$\sigma_{r}$ & 0.05 \\
$\mu_{l}$ & 25 \\
$\lambda$ & 0.5 \\
$a$ & 0.2 \\
$\rho$ & -0.1 \\
$\sigma_{l}$ & 20 \\
$\sigma_{s}$ & 0.2 \\
$b$ & 0.3 \\
$\mu$ & 0.01 \\
$H$ & 0.8 \\
\hline
\end{tabular}

correlation coefficients $\rho$, which illustrates that the option prices decrease when the correlation coefficients $\rho$ increase. By the light of nature, this is correct. The reason is if the asset price is strongly negatively correlated to the spot interest rates, the higher the asset price is, the lower the spot interest rates are and the higher the option price is. However, the changes in $\rho$ do not affect the option prices as significantly as changes in the volatility.

In Figure 3, we illustrate how the option prices are affected by the mean $\mu_{l}$ and standard deviation $\sigma_{l}$ of the claim size for two different trigger ratio levels of $c=1$ and $c=2$, respectively. For the trigger ratio level of $c=2$, the option price decreases as the mean of the claim sizes increases, while for the trigger ratio level of $c=1$, the option price increases as the mean of the claim sizes increases. This result is explained by exploring the risk-neutral probability in Figure 4(a) that the option is exercised. In Figure 4(a), the probability of exercising the option is an increasing function of the mean for smaller trigger ratio levels, while it is generally a constant function of the mean for larger trigger ratio levels. In addition, the probability of exercising the option for smaller trigger ratio levels is greater than that for larger ones. In Figure 3, we also find that, for the trigger ratio level of $c=2$, the option price increases as the standard deviation of the claim sizes increases, while for the trigger ratio level of $c=1$, the option price increases when the standard deviation of the claim sizes becomes smaller. However, after a certain value of $\sigma_{l}$ and then onward, the option price decreases as $\sigma_{l}$ increases. This reason is that, for reasonably small $\sigma_{l}$ and the trigger ratio level of $c$, the option price is dominated by the risk-neutral probability, which is monotonically decreasing with $\sigma_{l}$, as shown in Figure 4(b).

\section{Dynamically Hedging the CatEPut Option}

A reinsurance company provides a CatEPut option to an insurance company, and they need to hedge risk for this contract to avoid taking on very large losses. In this section, we present how to hedge against stock price risk and interest rate risk. In a perfect market, delta-gamma hedging techniques are used to measure the sensitivity of the option's price to underlying asset price movements at the first

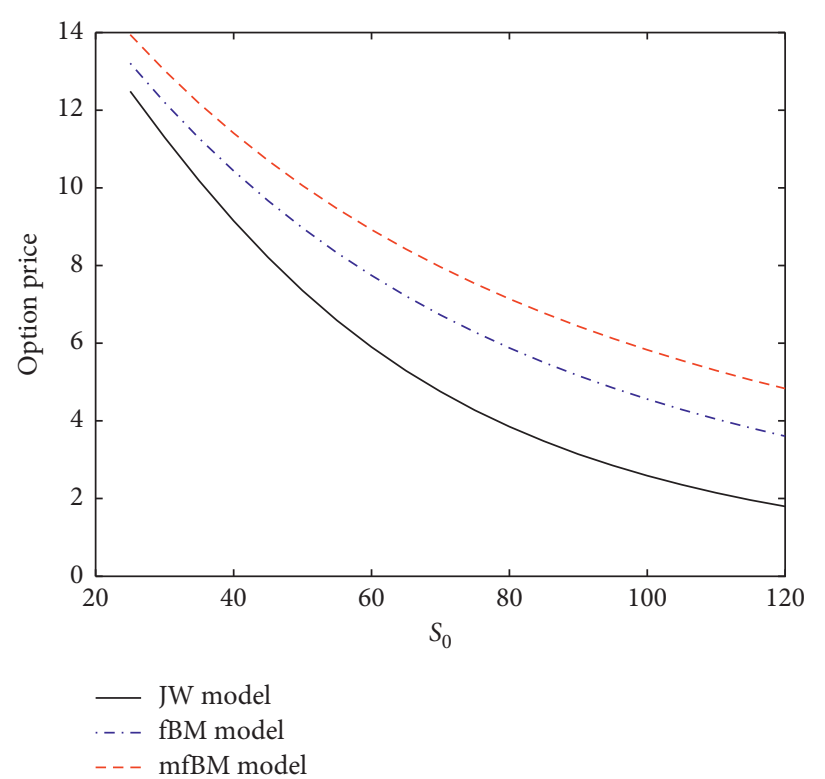

(a)

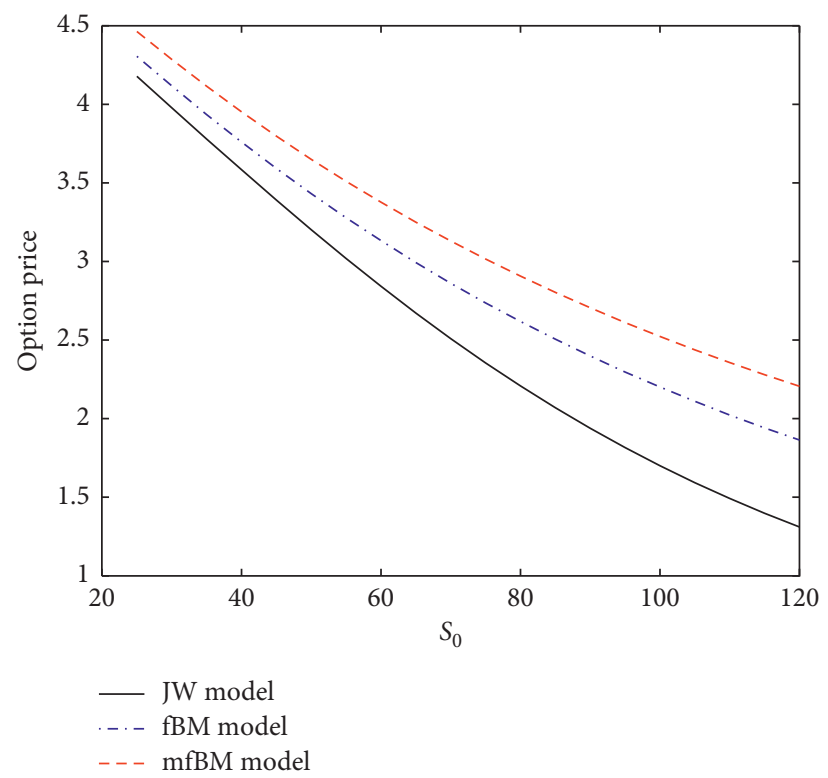

(b)

Figure 1: CatEPut option value for two trigger ratio levels $c=1$ and $c=2$ under the three specifications, JW, $\mathrm{fBM}$, and mfBM models. (a) $c=1$. (b) $c=2$.

and second order. Jaimungal and Wang [2] further used the delta-gamma-rho hedging strategy to measure the price risk of the stock price and interest rate. Here, we also use this technique to measure the price risk and compute various Greek values. The Greek letters are described as follows.

Proposition 4. Under the dynamics processes $S_{t}, L_{t}$, and $r_{t}$ modeled by (1) and (7), delta, gamma, and rho of the CatEPut option given in (17) are calculated as the following expressions: 
TABLE 2: Option prices under constant/stochastic interest rate.

\begin{tabular}{|c|c|c|c|c|c|c|}
\hline \multirow{2}{*}{$S_{0}$} & \multicolumn{3}{|c|}{ Constant interest rate } & \multicolumn{3}{|c|}{ Stochastic interest rate } \\
\hline & $c=1$ & $c=2$ & $c=3$ & $c=1$ & $c=2$ & $c=3$ \\
\hline \multicolumn{7}{|c|}{$H=0.8$} \\
\hline 60 & 17.7834 & 6.19015 & 1.45349 & 8.92275 & 3.37690 & 0.849746 \\
\hline 80 & 14.9291 & 5.56540 & 1.37039 & 7.14182 & 2.90722 & 0.776963 \\
\hline 100 & 12.6302 & 5.01053 & 1.29149 & 5.82774 & 2.52269 & 0.711669 \\
\hline \multicolumn{7}{|c|}{$H=0.9$} \\
\hline 60 & 18.3864 & 6.25808 & 1.45731 & 9.72182 & 3.53258 & 0.870091 \\
\hline 80 & 15.7647 & 5.67737 & 1.37779 & 8.03146 & 3.09770 & 0.802079 \\
\hline 100 & 13.6421 & 5.16633 & 1.30325 & 6.75448 & 2.73960 & 0.741522 \\
\hline \multicolumn{7}{|c|}{$H=0.98$} \\
\hline 60 & 18.9851 & 6.33379 & 1.46235 & 10.4918 & 3.68761 & 0.891346 \\
\hline 80 & 16.5656 & 5.79439 & 1.38677 & 8.88816 & 3.2833 & 0.827782 \\
\hline 100 & 14.5964 & 5.3226 & 1.31667 & 7.6544 & 2.94864 & 0.771475 \\
\hline \multicolumn{7}{|c|}{$\sigma_{s}=0.2$} \\
\hline 60 & 17.7834 & 6.19015 & 1.45349 & 8.92275 & 3.37690 & 0.849746 \\
\hline 80 & 14.9291 & 5.56540 & 1.37039 & 7.14182 & 2.90722 & 0.776963 \\
\hline 100 & 12.6302 & 5.01053 & 1.29149 & 5.82774 & 2.52269 & 0.711669 \\
\hline \multicolumn{7}{|c|}{$\sigma_{s}=0.25$} \\
\hline 60 & 18.9757 & 6.33639 & 1.46282 & 10.0168 & 3.55346 & 0.864323 \\
\hline 80 & 16.5352 & 5.79539 & 1.38735 & 8.43109 & 3.14982 & 0.800556 \\
\hline 100 & 14.5444 & 5.32101 & 1.31725 & 7.21853 & 2.81694 & 0.74415 \\
\hline \multicolumn{7}{|c|}{$\sigma_{s}=0.5$} \\
\hline 60 & 24.6637 & 7.27578 & 1.56092 & 14.8864 & 4.45694 & 0.969254 \\
\hline 80 & 23.5855 & 7.03926 & 1.52517 & 14.1160 & 4.27946 & 0.941041 \\
\hline 100 & 22.6716 & 6.83390 & 1.49338 & 13.4775 & 4.12875 & 0.916482 \\
\hline
\end{tabular}
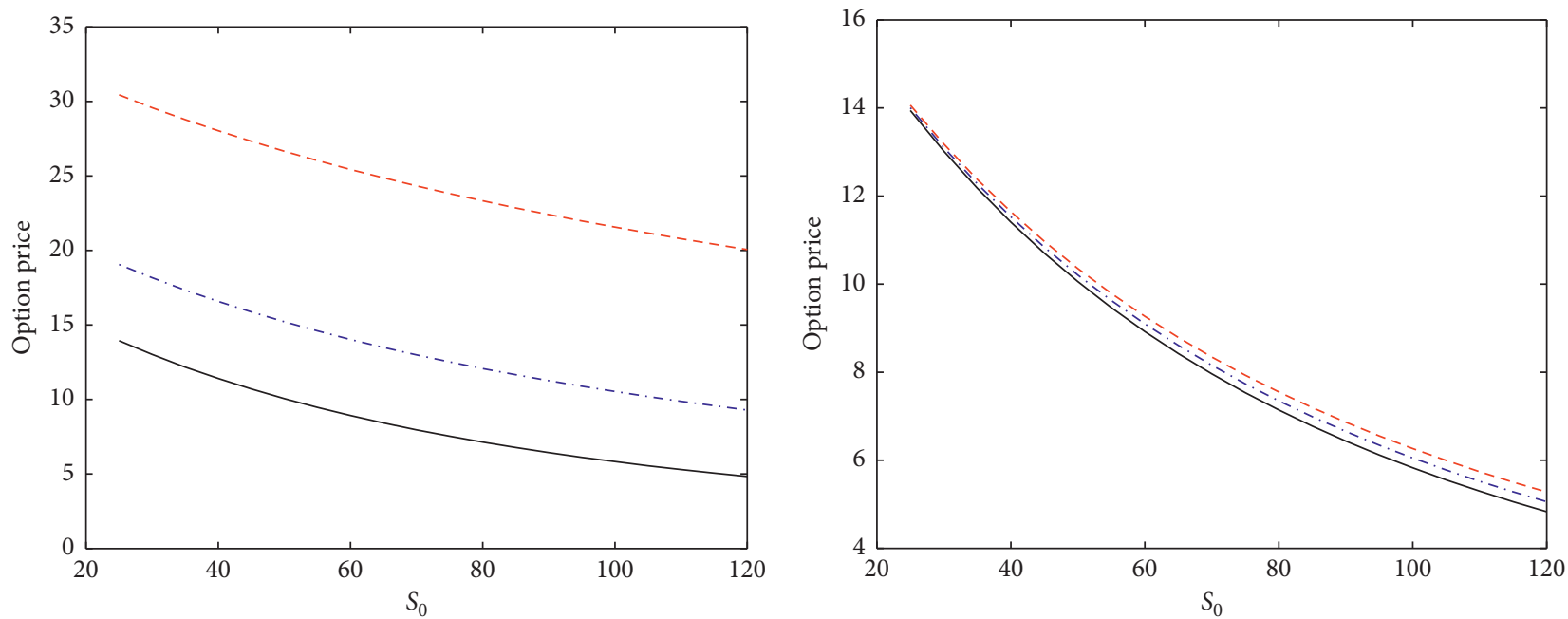

$$
\begin{aligned}
-\sigma_{\mathrm{r}} & =0.05 \\
--\sigma_{\mathrm{r}} & =0.1 \\
-- & \sigma_{\mathrm{r}}=0.15
\end{aligned}
$$$$
\begin{aligned}
-\rho & =-0.1 \\
--\rho & =0.0 \\
--\rho & =0.1
\end{aligned}
$$

(a)

(b)

FIGURE 2: Option price for several parameter values of the interest rate. (a) Volatility levels. (b) Correlation coefficients. 


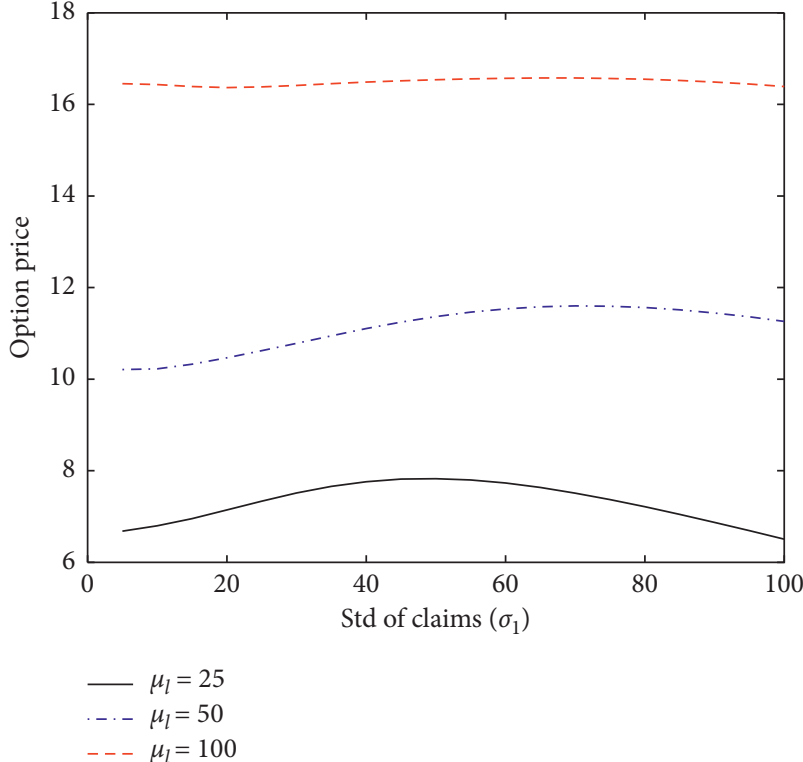

(a)

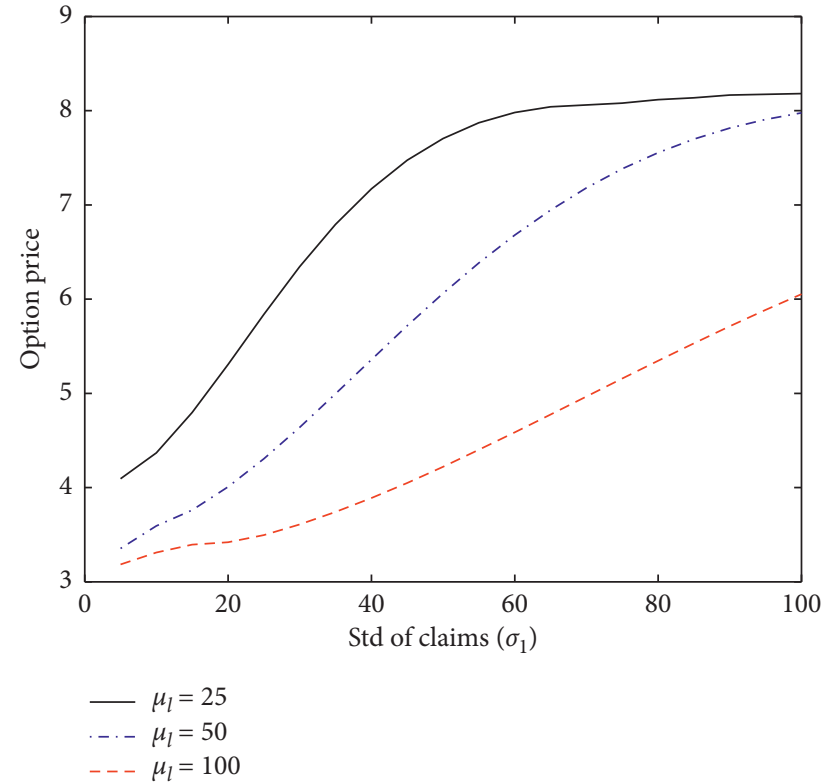

(b)

FIgURE 3: The effect of $\sigma_{l}$ on option price with different $\mu_{l}$. (a) $c=1$. (b) $c=2$.

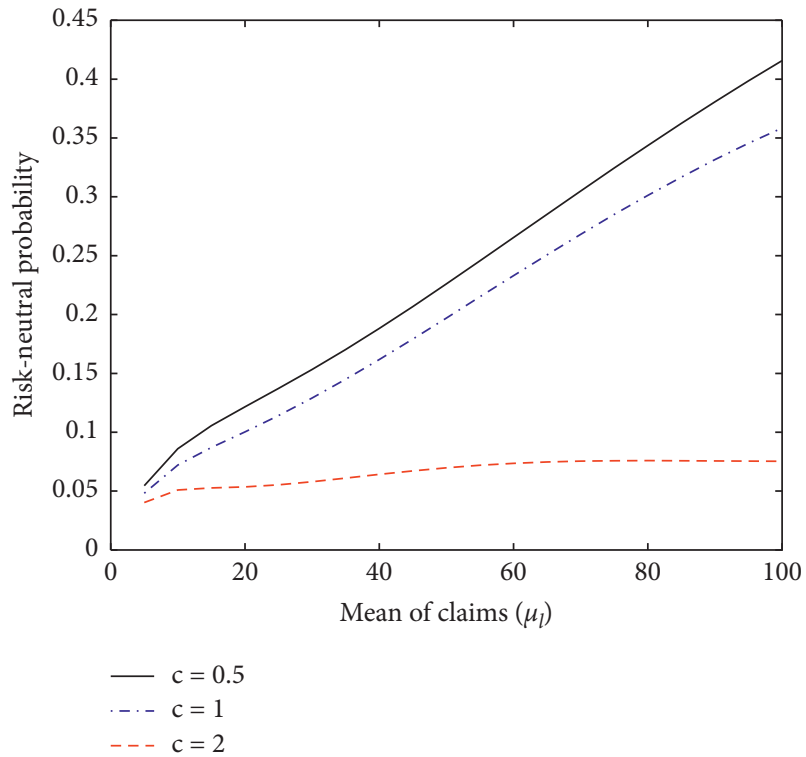

(a)

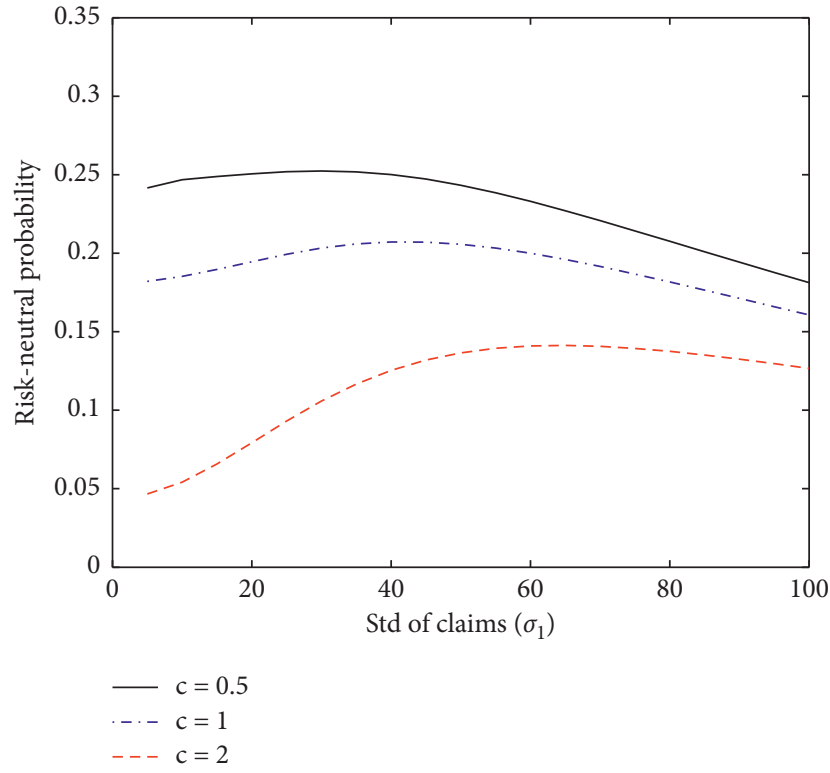

(b)

FIGURE 4: The effects of $\mu_{l}$ and $\sigma_{l}$ on risk-neutral probability of ending in-the-money for several trigger ratio levels of $c$. (a) Mean of claims $\mu_{l}$. (b) Std of claims $\sigma_{l}$. 
TABLE 3: Values of delta, gamma, and rho under JW and mfBM models.

\begin{tabular}{|c|c|c|c|c|c|c|}
\hline \multirow{2}{*}{$S_{0}$} & \multicolumn{3}{|c|}{ JW model } & \multicolumn{3}{|c|}{ mfBM model } \\
\hline & 60 & 80 & 100 & 60 & 80 & 100 \\
\hline \multicolumn{7}{|l|}{$\overline{c=1}$} \\
\hline$\Delta_{S}\left(t, t_{0}\right)$ & -0.12908 & -0.07965 & -0.05676 & -0.10417 & -0.07585 & -0.04934 \\
\hline$\Gamma_{S}\left(t, t_{0}\right)$ & 0.00303 & 0.00194 & 0.00116 & 0.00173 & 0.00115 & 0.00078 \\
\hline$\rho\left(t, t_{0}\right)$ & -72.3660 & -54.2121 & -39.8832 & -80.4577 & -70.0462 & -61.0020 \\
\hline \multicolumn{7}{|l|}{$c=2$} \\
\hline$\Delta_{S}\left(t, t_{0}\right)$ & -0.03472 & -0.02853 & -0.02229 & -0.02596 & -0.02119 & -0.01741 \\
\hline$\Gamma_{S}\left(t, t_{0}\right)$ & 0.00032 & 0.00029 & 0.00028 & 0.00027 & 0.00021 & 0.00017 \\
\hline$\rho\left(t, t_{0}\right)$ & -26.1142 & -23.8098 & -20.8363 & -26.1645 & -24.4051 & -22.6102 \\
\hline
\end{tabular}

$$
\begin{aligned}
\Delta_{S}\left(t, t_{0}\right)= & \frac{\partial P_{\mathrm{cat}}\left(t, t_{0}\right)}{\partial S_{t}}=-\sum_{n=1}^{\infty} \frac{[\lambda(T-t)]^{n} e^{\lambda(\kappa-1)(T-t)}}{n !} \\
& \cdot \int_{\tilde{L}}^{+\infty} e^{-\mu y} \Phi\left(-\widetilde{d}_{1}(y)\right) f_{Y}^{(n)}(y) \mathrm{d} y, \\
\Gamma_{S}\left(t, t_{0}\right)= & \frac{\partial^{2} P_{\mathrm{cat}}\left(t, t_{0}\right)}{\partial S_{t}^{2}}=\sum_{n=1}^{\infty} \frac{[\lambda(T-t)]^{n} e^{\lambda(\kappa-1)(T-t)}}{n !} \\
& \cdot \frac{1}{\sqrt{2 \pi} S_{t} \theta(t, T)} \int_{\tilde{L}}^{+\infty} e^{-\mu y-\widetilde{d}_{1}^{2}(y) / 2} f_{Y}^{(n)}(y) \mathrm{d} y, \\
\rho\left(t, t_{0}\right)= & \frac{\partial P_{\mathrm{cat}}\left(t, t_{0}\right)}{\partial r_{t}}=\frac{\partial P_{\mathrm{cat}}\left(t, t_{0}\right)}{\partial P(t, T)} \cdot \frac{\partial P(t, T)}{\partial r_{t}} \\
= & -K B(t, T) P(t, T) \cdot \sum_{n=1}^{\infty} \frac{[\lambda(T-t)]^{n} e^{\lambda(\kappa-1)(T-t)}}{n !} \\
& \int_{\tilde{L}}^{+\infty} \Phi\left(-\tilde{d}_{2}(y)\right) f_{Y}^{(n)}(y) \mathrm{d} y .
\end{aligned}
$$

Table 3 reports the values of delta, gamma, and rho for the in-the-money, at-the-money, and out-of-the-money CatEPut option under JW and mfBM models with two different trigger ratio levels of $c=1$ and $c=2$. First, the values of delta and gamma under the JW model are greater than those of the mfBM model. However, the values of rho under the JW model are smaller than those of the mfBM model. These findings are consistent with the results in Figure 1 since higher option's price leads to lower the values of delta and gamma and higher the values of rho.
Second, the values of delta, gamma, and rho for the CatEPut option decrease from in-the-money to out-of-themoney. Finally, the values of delta, gamma, and rho for the CatEPut option are sensitive to the trigger ratio level $c$ and are decreasing with the trigger ratio level $c$.

\section{Conclusion}

This paper extends the analysis of Jaimungal and Wang [2] to make it more realistic by introducing the $\mathrm{mfBM}$ model, which includes the $\mathrm{fBM}$ model and the BM model in the literature as its special cases, to represent the underlying asset price and interest rate changes. A closed-form analytical expression for the price of the CatEPut option is then derived in the case of deterministic and stochastic interest rates based on the fractional Girsanov formula. Finally, through numerical experiments, the quantitative impacts of different parameters on the option price and hedging parameters of the CatEPut option are discussed.

Some possible extensions should be considered in future research. Firstly, the proposed model can be tested empirically by using the catastrophic data from PCS (Property Claim Service). For example, we can estimate the parameters of catastrophe arrival rate $\lambda$ and the Hurst index $H$ using the maximum likelihood estimation method. Secondly, the proposed model can also be extended by taking the counterparty risk into account and evaluating other catastrophelinked financial options.

\section{Appendix}

\section{A. The Proof of Proposition 1}

Using the risk-neutral pricing theory and the law of iterated conditional expectation, we have

$$
\begin{aligned}
P_{\text {cat }}\left(t, t_{0}\right) & =E\left\{1_{\left(L_{T}-L_{t_{0}}>L\right)} E\left[e^{-\int_{t}^{T} r_{u} \mathrm{~d} u}\left(K-S_{T}\right)^{+} \mid L_{T}-L_{t}=\sum_{i=1}^{N_{T-t}} Y_{i}\right] \mid \mathscr{F}_{t}\right\} \\
& =I_{1}-I_{2},
\end{aligned}
$$


where

$$
\begin{aligned}
& I_{1}=K e^{-\int_{t}^{T} r_{u} \mathrm{~d} u} E\left\{1_{\left(L_{T}-L_{t}>L+L_{t_{0}}-L_{t}\right)} E\left[1_{\left(S_{T} \leq K\right)} \mid L_{T}-L_{t}=\sum_{i=1}^{N_{T-t}} Y_{i}\right] \mid \mathscr{F}_{t}\right\}
\end{aligned}
$$

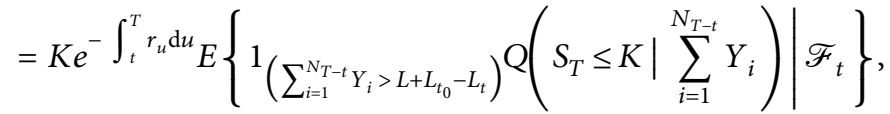

$$
\begin{aligned}
& I_{2}=E\left\{1\left(_{\left(L_{T}-L_{t}>L+L_{t_{0}}-L_{t}\right)} E\left[S_{T} e^{-\int_{t}^{T} r_{u} \mathrm{~d} u} 1_{\left(S_{T} \leq K\right)} \mid L_{T}-L_{t}=\sum_{i=1}^{N_{T-t}} Y_{i}\right] \mid \mathscr{F}_{t}\right\},\right. \\
& =E\left\{1\left(\sum_{i=1}^{\left.N_{T-t} Y_{i}>L+L_{t_{0}}-L_{t}\right)} E\left[S_{T} e^{-\int_{t}^{T} r_{u} \mathrm{~d} u} 1_{\left(S_{T} \leq K\right)} \mid \sum_{i=1}^{N_{T-t}} Y_{i}\right] \mid \mathscr{F}_{t}\right\}\right. \text {. }
\end{aligned}
$$

From (2), thus, formula $I_{1}$ in (A.2) can be rewritten as

$$
\begin{aligned}
& I_{1}=K e^{-\int_{t}^{T} r_{u} \mathrm{~d} u} \sum_{n=1}^{\infty} \frac{[\lambda(T-t)]^{n} e^{-\lambda(T-t)}}{n !} E\left\{1\left(\sum_{i=1}^{n} Y_{i}>L+L_{t_{0}}-L_{t}\right) \cdot Q\left(\ln S_{T} \leq \ln K \mid \sum_{i=1}^{n} Y_{i}\right) \mid \mathscr{F}_{t}\right\} \\
& =K e^{-\int_{t}^{T} r_{u} \mathrm{~d} u} \sum_{n=1}^{\infty} \frac{[\lambda(T-t)]^{n} e^{-\lambda(T-t)}}{n !} E\left\{1 _ { ( \sum _ { i = 1 } ^ { n } Y _ { i } > L + L _ { t _ { 0 } } - L _ { t } ) } \cdot \mathcal { Q } \left(\sigma_{s}\left(M_{T}^{H}-M_{t}^{H}\right)\right.\right. \\
& \left.\left.\leq-\left[\ln \frac{S_{t}}{K}-\mu \sum_{i=1}^{n} Y_{i}+\lambda \mathcal{K}(T-t)+\int_{t}^{T} r_{u} \mathrm{~d} u-\frac{1}{2} \sigma_{s}^{2}\left[\alpha^{2}(T-t)+\beta^{2}\left(T^{2 H}-t^{2 H}\right)\right]\right] \mid \sum_{i=1}^{n} Y_{i}\right) \mid \mathscr{F}_{t}\right\} \\
& =\sum_{n=1}^{\infty} \frac{[\lambda(T-t)]^{n} e^{-\lambda(T-t)}}{n !} \int_{\widetilde{L}}^{+\infty} K e^{-\int_{t}^{T} r_{u} \mathrm{~d} u} \Phi\left(-d_{2}(y)\right) f_{Y}^{(n)}(y) \mathrm{d} y .
\end{aligned}
$$

Similarly, we rewrite (A.3) as

$$
\begin{aligned}
I_{2} & =S_{t} E\left\{1\left(\sum_{i=1}^{\left.N_{T-t} Y_{i}>L+L_{t_{0}}-L_{t}\right)} E\left[\frac{S_{T} e^{-\int_{t}^{T} r_{u} \mathrm{~d} u}}{S_{t}} 1_{\left(S_{T} \leq K\right)} \mid \sum_{i=1}^{N_{T-t}} Y_{i}\right] \mid \mathscr{F}_{t}\right\}\right. \\
& =S_{t} \widetilde{E}\left\{1\left(\sum_{i=1}^{N_{T-t} Y_{i}>L+L_{t_{0}}-L_{t}}\right) \widetilde{Q}\left(\ln S_{T} \leq \ln K \mid \sum_{i=1}^{N_{T-t}} Y_{i}\right) \mid \mathscr{F}_{t}\right\},
\end{aligned}
$$

where $\widetilde{E}\left[\mathscr{F}_{t}\right]$ denotes the quasi-conditional expectation with respect to the probability measure $\widetilde{Q}$ which is equivalent to $Q$, and the Radon-Nikodym derivative which induced the measure change from $Q$ to $\widetilde{Q}$ is given by the formula

$$
\left.\frac{\mathrm{d} \widetilde{Q}}{\mathrm{dQ}}\right|_{\mathscr{F}_{T}}=\frac{S_{T} e^{-\int_{0}^{T} r_{u} \mathrm{~d} u}}{S_{0}}=\exp \left\{-\frac{1}{2}\left[\alpha^{2} T+\beta^{2} T^{2 H}\right]+\sigma_{s} M_{T}^{H}\right\} .
$$

Let $\widetilde{M}_{t}^{H}=\alpha \widetilde{W}_{t}+\beta \widetilde{W}_{t}^{H}=M_{t}^{H}-\sigma_{s}\left(\alpha^{2} t+\beta^{2} t^{2 H}\right)$; $\begin{gathered}\text { using } \\ \text { using }\end{gathered}$ the fractional Girsanov theorem (see Hu and Oksendal [45]), then $\widetilde{M}_{t}^{H}$ is also a mfBM with parameters $\alpha, \beta$, and $H$ under the new probability measure $\widetilde{Q}$. Hence, we can rewrite (2) as

$$
\begin{aligned}
\ln S_{T}= & \ln S_{t}+\left\{\int_{t}^{T} r_{u} \mathrm{~d} u-\mu\left(L_{T}-L_{t}\right)+\lambda \kappa(T-t)\right. \\
& \left.+\frac{1}{2} \sigma_{s}^{2}\left[\alpha^{2}(T-t)+\beta^{2}\left(T^{2 H}-t^{2 H}\right)\right]+\sigma_{s}\left(\tilde{M}_{T}^{H}-\tilde{M}_{t}^{H}\right)\right\} .
\end{aligned}
$$

under $\widetilde{Q}$ and obtain that 


$$
\begin{aligned}
I_{2} & =S_{t} \sum_{n=1}^{\infty} \frac{[\lambda(T-t)]^{n} e^{-\lambda(T-t)}}{n !} E\left\{1 ( \sum _ { i = 1 } ^ { n } Y _ { i } > L + L _ { t } - L _ { t } ) e ^ { - \mu \sum _ { i = 1 } ^ { n } Y _ { i } + \lambda \kappa ( T - t ) } \cdot \tilde { Q } \left(\sigma_{s}\left(M_{T}^{H}-M_{t}^{H}\right)\right.\right. \\
& \left.\left.\leq-\left[\ln \frac{S_{t}}{K}-\mu \sum_{i=1}^{n} Y_{i}+\lambda \kappa(T-t)+\int_{t}^{T} r_{u} \mathrm{~d} u \frac{1}{2} \sigma_{s}^{2}\left[\alpha^{2}(T-t)+\beta^{2}\left(T^{2 H}-t^{2 H}\right)\right]\right] \mid \sum_{i=1}^{n} Y_{i}\right) \mid \mathscr{F}_{t}\right\} \\
& =\sum_{n=1}^{\infty} \frac{[\lambda(T-t)]^{n} e^{-\lambda(T-t)}}{n !} \int_{\tilde{L}}^{+\infty} S_{t} e^{-\mu y+\lambda \kappa(T-t)} \Phi\left(-d_{1}(y)\right) f_{Y}^{(n)}(y) \mathrm{d} y .
\end{aligned}
$$

From (A.4) and (A.8), the proof is completed.

\section{B. The Proof of Proposition 2}

From (16), we write $P_{\text {cat }}\left(t, t_{0}\right)$ as a sum of two terms $\widehat{I}_{1}$ and $\widehat{I}_{2}$ :

$$
\begin{aligned}
& \widehat{I}_{1}=K P(t, T) E^{T}\left[{ }_{\left(L_{T}-L_{t}>L+L_{t_{0}}-L_{t}\right)}{ }^{1}\left(\ln S_{T} \leq \ln K\right) \mid \mathscr{F}_{t}\right], \\
& \widehat{I}_{2}=P(t, T) E^{T}\left[1_{\left(L_{T}-L_{t}>L+L_{t_{0}}-L_{t}\right)} S_{T} 1_{\left(\ln S_{T} \leq \ln K\right)} \mid \mathscr{F}_{t}\right] .
\end{aligned}
$$

$$
\begin{aligned}
& \widehat{I}_{1}=K P(t, T) E^{T}\left[1_{\left(L_{T}-L_{t}>L+L_{t_{0}}-L_{t}\right)}{ }^{1}\left(\ln S_{T} \leq \ln K\right) \mid \mathscr{F}_{t}\right] \\
& =K P(t, T) E^{T}\left[1_{\left(L_{T}-L_{t}>L+L_{t_{0}}-L_{t}\right)} E^{T}\left\{1_{\left(\ln S_{T} \leq \ln K\right)} \mid L_{T}-L_{t}\right\} \mid \mathscr{F}_{t}\right] \\
& =K P(t, T) \sum_{n=1}^{\infty} \frac{[\lambda(T-t)]^{n} e^{-\lambda(T-t)}}{n !} E\left\{1_{\left(\sum_{i=1}^{n} Y_{i}>L+L_{t_{0}}-L_{t}\right)} \cdot Q^{T}\left(\ln S_{T} \leq \ln K \mid \sum_{i=1}^{n} Y_{i}\right) \mid \mathscr{F}_{t}\right\} \\
& =K P(t, T) \sum_{n=1}^{\infty} \frac{[\lambda(T-t)]^{n} e^{-\lambda(T-t)}}{n !} E\left\{1 \sum_{i=1}^{n} Y_{i}>L+L_{t_{0}}-L_{t} \cdot Q^{T}\left(\int_{t}^{T} \sigma_{s} \mathrm{~d} \widehat{M}_{t}^{H}+\int_{t}^{T} \sigma_{r} B(u, T) \mathrm{d} \widehat{Z}_{t}^{H} \leq \gamma \mid \sum_{i=1}^{n} Y_{i}\right) \mid \mathscr{F}_{t}\right\} \\
& =\sum_{n=1}^{\infty} \frac{[\lambda(T-t)]^{n} e^{-\lambda(T-t)}}{n !} \int_{\widetilde{L}}^{+\infty} K P(t, T) \Phi\left(-\widehat{d}_{2}(y)\right) f_{Y}^{(n)}(y) \mathrm{d} y .
\end{aligned}
$$

We denote $\gamma=-\left[\ln \left(S_{t} / K P(t, T)\right)-\mu \sum_{i=1}^{n} Y_{i}+\lambda \kappa(T-\right.$ $\left.t)-(1 / 2) \theta^{2}(t, T)\right]$ and firstly calculate the term $\widehat{I}_{1}$. By using (14), we have

Similarly, we have

$$
\begin{aligned}
\widehat{I}_{2}= & P(t, T) E^{T}\left[{ }_{\left(L_{T}-L_{t}>L+L_{t_{0}}-L_{t}\right)} S_{T} 1_{\left.\left(\ln S_{T} \leq \ln K\right) \mid \mathscr{F}_{t}\right]}\right. \\
= & P(t, T) E^{T}\left[{ } _ { ( L _ { T } - L _ { t } > L + L _ { t _ { 0 } } - L _ { t } ) } E ^ { T } \left\{e ^ { \operatorname { l n } S _ { T } 1 } 1 _ { ( \operatorname { l n } S _ { T } \leq \operatorname { l n } K ) | L _ { T } - L _ { t } \} | \mathscr { F } _ { t } ] } E ^ { T } \left\{1\left(\sum_{i=1}^{n} Y_{i}>L+L_{t_{0}}-L_{t}\right) e^{-\mu \sum_{i=1}^{n} Y_{i}+\lambda \kappa(T-t)-(1 / 2) \theta^{2}(t, T)} \cdot E^{T}\right.\right.\right. \\
= & S_{t} \sum_{n=1}^{\infty} \frac{[\lambda(T-t)]^{n} e^{-\lambda(T-t)}}{n !} \\
& \left.\left.\cdot\left[e^{\int_{t}^{T} \sigma_{s} \mathrm{~d} \widehat{M}_{t}^{H}+\int_{t}^{T} \sigma_{r} B(u, T) \mathrm{d} \widehat{Z}_{t}^{H}} 1 \int_{t}^{T} \sigma_{s} \mathrm{~d} \widehat{M}_{t}^{H}+\int_{t}^{T} \sigma_{r} B(u, T) \mathrm{d} \widehat{Z}_{t}^{H} \leq \gamma\right) \mid \sum_{i=1}^{n} Y_{i}\right] \mid \mathscr{F}_{t}\right\} .
\end{aligned}
$$


Using the fact that $E\left[e^{X} 1_{(X<\gamma)}\right]=e^{\delta^{2} / 2} \Phi((\gamma+\delta) / \delta)$ when $X \sim N\left(0, \delta^{2}\right)$, then

$$
\begin{aligned}
& \widehat{I}_{2}=S_{t} \sum_{n=1}^{\infty} \frac{[\lambda(T-t)]^{n} e^{-\lambda(T-t)}}{n !} E^{T}\left\{1\left(\sum_{i=1}^{n} Y_{i}>L+L_{t_{0}}-L_{t}\right)\right. \\
&\left.\cdot e^{-\mu \sum_{i=1}^{n} Y_{i}+\lambda \kappa(T-t)} \Phi\left(\frac{\gamma+\theta(t, T)}{\theta(t, T)}\right) \mid \mathscr{F}_{t}\right\} \\
&=\sum_{n=1}^{\infty} \frac{[\lambda(T-t)]^{n} e^{-\lambda(T-t)}}{n !} \int_{\tilde{L}}^{+\infty} S_{t} e^{-\mu y+\lambda \kappa(T-t)} \Phi\left(-\widehat{d}_{2}(y)\right) f_{Y}^{(n)}(y) \mathrm{d} y .
\end{aligned}
$$

Hence, (B.2) and (B.4) yield (17).

\section{Data Availability}

All data used to support the findings of this study are included within this article.

\section{Conflicts of Interest}

The author declares that there are no conflicts of interest regarding the publication of this paper.

\section{Acknowledgments}

This research was supported by the NSF of China (grant no. 11461008) and the Guangxi Natural Science Foundation (grant no. 2018GXNSFAA281016).

\section{References}

[1] S. H. Cox, J. R. Fairchild, and H. W. Pedersen, "Valuation of structured risk management products," Insurance: Mathematics and Economics, vol. 34, no. 2, pp. 259-272, 2004.

[2] S. Jaimungal and T. Wang, "Catastrophe options with stochastic interest rates and compound Poisson losses," Insurance: Mathematics and Economics, vol. 38, no. 3, pp. 469-483, 2006.

[3] O. Vasicek, "An equilibrium characterization of the term structure," Journal of Financial Economics, vol. 5, no. 2, pp. 177-188, 1977.

[4] L.-F. Chang and M.-W. Hung, "Analytical valuation of catastrophe equity options with negative exponential jumps," Insurance: Mathematics and Economics, vol. 44, no. 1, pp. 59-69, 2009.

[5] S.-K. Lin, C.-C. Chang, and M. R. Powers, "The valuation of contingent capital with catastrophe risks," Insurance: Mathematics and Economics, vol. 45, no. 1, pp. 65-73, 2009.

[6] X. S. Lin and T. Wang, "Pricing perpetual American catastrophe put options: a penalty function approach," Insurance: Mathematics and Economics, vol. 44, no. 2, pp. 287-295, 2009.

[7] I.-M. Jiang, S.-Y. Yang, Y.-H. Liu, and A. T. Wang, "Valuation of double trigger catastrophe options with counterparty risk," The North American Journal of Economics and Finance, vol. 25, pp. 226-242, 2013.

[8] Y. Jin and S. Zhong, "New explicit closed form formulae for the prices of catastrophe options," International Journal of Financial Engineering, vol. 2, pp. 1-16, 2015.
[9] E. Koo and G. Kim, "Explicit formula for the valuation of catastrophe put option with exponential jump and default risk," Chaos, Solitons \& Fractals, vol. 101, pp. 1-7, 2017.

[10] Y. Xu and G. Wang, "Pricing catastrophe options with counterparty credit risk in a reduced form model," Acta Mathematica Scientia, vol. 38, no. 1, pp. 347-360, 2018.

[11] H.-S. Kim, B. Kim, and J. Kim, "Catastrophe equity put options under stochastic volatility and catastrophe-dependent jumps," Journal of Industrial \& Management Optimization, vol. 10, no. 1, pp. 41-55, 2014.

[12] S. L. Heston, "A closed-form solution for options with stochastic volatility with applications to bond and currency options," Review of Financial Studies, vol. 6, no. 2, pp. 327343, 1993.

[13] X. Wang, "Catastrophe equity put options with target variance," Insurance: Mathematics and Economics, vol. 71, pp. 79-86, 2016.

[14] W. Wang, L. Qian, and X. Su, "Pricing and hedging catastrophe equity put options under a Markov-modulated jump diffusion model," Journal of Industrial \& Management Optimization, vol. 11, no. 2, pp. 493-514, 2015.

[15] H.-S. Kim, B. Kim, and J. Kim, "Pricing perpetual American CatEPut options when stock prices are correlated with catastrophe losses," Economic Modelling, vol. 41, pp. 15-22, 2014b.

[16] J. Yu, "Catastrophe options with double compound Poisson processes," Economic Modelling, vol. 50, pp. 291-297, 2015.

[17] W. Yu, P. Guo, Q. Wang et al., "On a periodic capital injection and barrier dividend strategy in the compound Poisson risk model," Mathematics, vol. 8, no. 4, pp. 511-531, 2020.

[18] W. Yu, Y. Yong, G. Guan, Y. Huang, W. Su, and C. Cui, "Valuing guaranteed minimum death benefits by cosine series expansion," Mathematics, vol. 7, no. 9, pp. 835-849, 2019.

[19] W. Yu, F. Wang, Y. Huang, and H. Liu, "Social optimal mean field control problem for population growth model," Asian Journal of Control, vol. 21, no. 4, pp. 1-8, 2019.

[20] Z. Zhang, Y. Yong, and W. Yu, "Valuing equity-linked death benefits in general exponential Lévy models," Journal of Computational and Applied Mathematics, vol. 365, Article ID 112377, 2020.

[21] A. W. Lo, "Long-term memory in stock market prices," Econometrica, vol. 59, no. 5, pp. 1279-1313, 1991.

[22] W. Willinger, M. S. Taqqu, and V. Teverovsky, "Stock market prices and long-range dependence," Finance and Stochastics, vol. 3, no. 1, pp. 1-13, 1999.

[23] R. Cont, "Empirical properties of asset returns: stylized facts and statistical issues," Quantitative Finance, vol. 1, no. 2, pp. 223-236, 2001.

[24] S. H. Kang and S.-M. Yoon, "Long memory features in the high frequency data of the Korean stock market," Physica A: 
Statistical Mechanics and Its Applications, vol. 387, no. 21, pp. 5189-5196, 2008.

[25] S. H. Kang, C. Cheong, and S.-M. Yoon, "Long memory volatility in Chinese stock markets," Physica A: Statistical Mechanics and Its Applications, vol. 389, no. 7, pp. 1425-1433, 2010.

[26] T.-C. Huang, Y.-C. Tu, and H.-C. Chou, "Long memory and the relation between options and stock prices," Finance Research Letters, vol. 12, pp. 77-91, 2015.

[27] Y. A. Kuznetsov, "The absence of arbitrage in a model with fractal Brownian motion," Russian Mathematical Surveys, vol. 54, no. 4, pp. 847-848, 1999.

[28] T. Bjork and H. Hult, "A note on wick products and the fractional Black-Scholes model," Finance and Stochastics, vol. 9, no. 2, pp. 197-209, 2005.

[29] P. Cheridito, "Mixed fractional Brownian motion," Bernoulli, vol. 7, no. 6, pp. 913-934, 2001.

[30] P. Cheridito, "Arbitrage in fractional Brownian motion models," Finance and Stochastics, vol. 7, no. 4, pp. 533-553, 2003.

[31] G. Deng and H. Xi, "Pricing reset option in a fractional Brownian motion market," in Proceedings of the 30th Chinese Control Conference, pp. 5727-5731, Yantai, China, July 2011.

[32] B. L. S. Prakasa Rao, "Pricing geometric Asian power options under mixed fractional Brownian motion environment," Physica A: Statistical Mechanics and Its Applications, vol. 446, pp. 92-99, 2016.

[33] L. Sun, "Pricing currency options in the mixed fractional Brownian motion," Physica A: Statistical Mechanics and Its Applications, vol. 392, no. 16, pp. 3441-3458, 2013.

[34] W.-L. Xiao, W.-G. Zhang, X. Zhang, and X. Zhang, "Pricing model for equity warrants in a mixed fractional Brownian environment and its algorithm," Physica A: Statistical Mechanics and Its Applications, vol. 391, no. 24, pp. 6418-6431, 2012.

[35] W. Xiao, W. Zhang, X. Zhang, and X. Chen, "The valuation of equity warrants under the fractional Vasicek process of the short-term interest rate," Physica A: Statistical Mechanics and Its Applications, vol. 394, pp. 320-337, 2014.

[36] W.-G. Zhang, Z. Li, and Y.-J. Liu, "Analytical pricing of geometric Asian power options on an underlying driven by a mixed fractional Brownian motion," Physica A: Statistical Mechanics and Its Applications, vol. 490, pp. 402-418, 2018.

[37] X. He and W. Chen, "The pricing of credit default swaps under a generalized mixed fractional Brownian motion," Physica A: Statistical Mechanics and Its Applications, vol. 404, pp. 26-33, 2014.

[38] F. Mehrdoust, A. R. Najafi, S. Fallah, and O. Samimi, "Mixed fractional Heston model and the pricing of American options," Journal of Computational and Applied Mathematics, vol. 330, pp. 141-154, 2018.

[39] J. McCarthy, R. DiSario, H. Saraoglu, and H. Li, "Tests of longrange dependence in interest rates using wavelets," The Quarterly Review of Economics and Finance, vol. 44, pp. 180-189, 2004.

[40] L. A. Gil-Alana, "Long memory in the interest rates in some Asian countries," International Advances in Economic Research, vol. 9, no. 4, pp. 257-267, 2003.

[41] B. M. Tabak and D. O. Cajueiro, "The long-range dependence behavior of the term structure of interest rates in Japan," Physica A: Statistical Mechanics and Its Applications, vol. 350, no. 2-4, pp. 418-426, 2005.

[42] D. O. Cajueiro and B. M. Tabak, "Long-range dependence and multifractality in the term structure of LIBOR interest rates,"
Physica A: Statistical Mechanics and Its Applications, vol. 373, pp. 603-614, 2007.

[43] D. O. Cajueiro and B. M. Tabak, "Testing for long-range dependence in the Brazilian term structure of interest rates," Chaos, Solitons \& Fractals, vol. 40, no. 4, pp. 1559-1573, 2009.

[44] Q. Zhou, Q. Wang, and W. Wu, "Pricing of defaultable securities associated with recovery rate under the stochastic interest rate driven by fractional Brownian motion," Journal of Systems Science and Complexity, vol. 32, no. 2, pp. 657-680, 2019.

[45] Y. Hu and B. Øksendal, "Fractional white noise calculus and applications to finance," Infinite Dimensional Analysis, Quantum Probability and Related Topics, vol. 6, no. 1, pp. 132, 2003. 